\title{
Studies by the U.S. Geological Survey in Alaska, 2001
}

Professional Paper 1678

U.S. Department of the Interior U.S. Geological Survey 
